Chirurg 2020 $91: 179-180$

https://doi.org/10.1007/s00104-020-01117-7

(C) Springer Medizin Verlag GmbH, ein Teil von Springer Nature 2020

\title{
Thomas Schmitz-Rixen
}

Gefäß- und Endovaskularchirurgie, Universitätsklinikum Frankfurt, Frankfurt am Main, Deutschland

\section{Intelligenz, Intuition und Individualität}

Was erklären wir nicht alles mit wechselnder Begeisterung zum Megatrend - Globalisierung, Klimawandel, Urbanisierung und in der Medizin Zentralisierung komplexer Leistungen. Mit wachsender Häufigkeit fällt auch der Begriff der Digitalisierung, die als nächste Industrialisierungsstufe mit exponentieller Kraft ein erhebliches disruptives Potenzial in unseren ökonomischen und sozialen Systemen erzeugt. Dies macht vor der Medizin und im Besonderen vor der seit jeher technikaffinen Chirurgie nicht halt. Welche Trends hält die Digitalisierungswelle für uns bereit und bedürfen einer näheren Betrachtung:

- Internet of Things (IoT): Mobile und stationäre Gegenstände werden durch Mikrochips smart und interagieren ohne menschliches Zutun mit anderen Objekten oder Computern.

- Logistics: die autonome Koordination von Güterströmen, Individualverkehr und lokalen Dienstleistungen.

- E-Commerce: grenzen- und zeitlose Beschaffung von Gütern, zunehmende Konkurrenz des stationären Handels.

- Smart Systems: Minitarisierung und Integration erzeugen komplexe und immer anspruchsvollere Anwendungen, Chancen auch in der Medizintechnik für Diagnostik und Therapie.

- Virtual Reality: Der Weg zum digitalen Zwilling mit dem Sinn der Therapieplanung und -erprobung, Voraussetzung für eine personalisierte Medizin im chirurgischen Umfeld.

- Social Media: Merkmal ist die Interaktivität, heute die bevorzugte Quelle der schnellen Informationsbeschaffung.
- Smart Infrastructure: intelligente Vernetzung mit nachhaltigem Umgang mit nicht erneuerbaren Ressourcen, "smart cities" entstehen zurzeit an 2 Standorten.

- Industrie 4.0: vierte industrielle ITgesteuerte Revolution zur Optimierung und Steuerung der Wertschöpfung.

- Big Data: Rohstoff der Zukunft, hieraus erzeugen selbstlernende Algorithmen „smart data“ (u.a. durch ,deep learning“).

- Künstliche Intelligenz (KI): selbstlernende Computersysteme mit selbständiger Entscheidungsfindung, erhebliches ethisches Spannungsfeld: Was können Maschinen besser als Menschen? Sind sie supportiv und assistierend oder autonom ersetzend?

- Cybersecurity: Schutz vor unautorisiertem Zugriff.

Parallel zum Deutschen Chirurgenkongress 2020 legen Beauftragte aller chirurgischen Fachgesellschaften und des Sanitätscorps der Bundeswehr den Stand der Implementierung digitaler Anwendungen und speziell künstlicher Intelligenz (KI) in ihren jeweiligen Fachgebieten dar. Die anfängliche Befürchtung einer Redundanz ist durch das Ergebnis sachgerecht zerstreut. Das sich bietende Bild ist bunt und weit gefächert und zeigt die Innovationskraft und den Willen der beteiligten Arbeitsgruppen, qualitativ hochwertige Anwendungen zu entwickeln und/oder zu implementieren. Vorwiegend kommerziell ausgerichtete milliardenschwere Investitionen in den USA und China lassen sich nicht toppen; umso mehr wird es unsere Aufgabe und unsere Chance sein, qualitativ hochwertige KIAnwendungen nutzbar zu machen und/ 
oder zu bewerten. Die Sicherheit der uns anvertrauten Daten muss hierbei eine hohe Priorität besitzen.

Auch ist die Thematik mit vielen falschen Erwartungen, Ängsten und heftigen Emotionen verknüpft, sodass es Zeit für eine Standortbestimmung wird. Daraus resultiert auch die Mottowahl des Kongresses: „Intelligenz, Intuition und Individualität“.

Gleich am Anfang gibt es eine Entwarnung für all diejenigen unter uns und dies dürften die meisten sein, die zurecht stolz aufihrehandwerklichen Fähigkeiten sind und diese auch zur Perfektion entwickelt haben. Nach Einschätzung der führenden KI-Experten wird es noch Jahrzehnte dauern, bis KI in der Lage ist, die komplexe Interaktion zwischen Gehirn und unseren trainierten Händen nachzubilden; dies gilt für komplexe kognitive Funktionen und die komplexe feinfühlige Motorik unserer Hände. So lange ist die Erwartungshaltung an KI eine Verbesserung der Patientensicherheit und bessere Langzeitergebnisse durch ideale Unterstützungssysteme für eine personalisierte Medizin.

Möge das vorliegende Heft eine profunde Informationsquelle für den jetzigen Stand der KI-Anwendungen vor allen Dingen für den digitalen Newcomer sein. Es ist nicht zu erwarten, dass die dargelegten Informationen lange up to date bleiben.

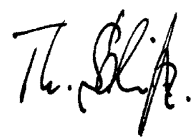

Prof. Dr. Thomas Schmitz-Rixen

\section{Korrespondenzadresse}

\section{Prof. Dr. Thomas Schmitz-Rixen}

Gefäß- und Endovaskularchirurgie,

Universitätsklinikum Frankfurt

Theodor-Stern-Kai 7, 60590 Frankfurt am Main, Deutschland

schmitz-rixen@em.uni-frankfurt.de

Interessenkonflikt. T. Schmitz-Rixen gibt an, dass kein Interessenkonflikt besteht.
Weißer, C.

\section{Chirurgenlexikon}

2000 Persönlichkeiten aus der Geschichte der Chirurgie

Berlin Heidelberg: Springer-Verlag 2019, 1. Auflage, 501 S., 135 Abb., (ISBN: 978-3-662-59237-3), Hardcover 99,99 EUR

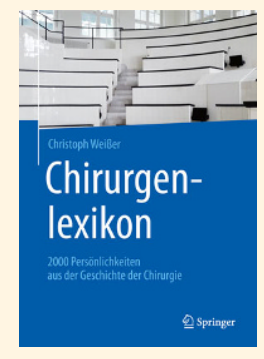

Beschäftigt man sich intensiver mit chirurgischen oder orthopädischen Fragestellungen, so bestätigt sich immer wieder das Gleichnis von den „Zwergen die auf den Schul-

tern von Riesen" sitzen. So rasch sich Medizin und Chirurgie gegenwärtig fortentwickeln, so sehr sind sie das Ergebnis eines jahrhundertelangen Erkenntnisprozesses. Die aktuelle chirurgische und orthopädische Praxis ist ohne die

die wissenschaftlichen Erkenntnisse und Erfahrungen früherer Chirurgengenerationen nicht zu verstehen.

Es ist das Verdienst des Würzburger Chirurgen und Oberarztes Christoph Weißer, ein umfassendes Lexikon zu wichtigen Persönlichkeiten der Chirurgie, Orthopädie und angrenzender Fachgebiete vorgelegt zu haben.

Der Autor informiert über die Lebensdaten, den beruflichen Werdegang und die Schwerpunkte der beruflichen Tätigkeit bedeutender Chirurgen. Weißer gelingt es dabei, die wesentlichen biographischen Aspekte knapp und verständlich darzustellen. Die Texte sind wissenschaftlich präzise, sie halten einer historischen Überprüfung stand und gehen weit über diejenigen Informationen hinaus, die bei einer Internetrecherche gewonnen werden können. Als Nachschlagewerk konzipiert, lädt es darüber hinaus zu einer spanenden und lehrreichen Lektüre ein. Wer wüsste nicht gerne mehr über Percival Pott, Bernhard Langenbeck, William A. Lane, Themistokles Gluck, Martin Kirschner, Albin Lambotte, K. H. Bauer, Martin Allgöwer und Klaus Klemm?

Der Autor erklärt die hauptsächlichen chirurgischen Eponyme und trägt somit zum
Verständnis von Begriffen bei, die zwar allgemein gebräuchlich sind, deren Kenntnis sich jedoch nicht unmittelbar erschließt. Weit über einhundert Abbildungen, zeitgenössische Zeichnungen und Fotografien, illustrieren den Band. Das Werk berücksichtigt einzelne Ärzte aus Antike und frühen Neuzeit, der Schwerpunkt liegt in dem Zeitraum vom 18. Jahrhundert bis in die Gegenwart. Berücksichtigt wurden die Lehrstuhlinhaber der chirurgischen Fächer im deutschsprachigen Raum, die Leiter der berufsgenossenschaftlichen Unfallkliniken sowie die Präsidenten der verschiedenen chirurgischen Fachgesellschaften.

Das umfassende Werk kann jedem Chirurgen und Orthopäden mit Nachdruck empfohlen werden.

K.-D. Thomann (Frankfurt am Main) 